\title{
Design and Experiment of Adaptive Anti-saturation and Anti-jamming Modules for GPS Receiver Based on 4-antenna Array
}

\author{
Jian-feng TAN ${ }^{1}$, Run-dong ZHENG ${ }^{2}$, Ming-tuan LIN ${ }^{3}$, Pei-guo LIU ${ }^{4}$ \\ and Ji-bin LIU ${ }^{5}$
}

College of Electronics Science and Engineering, National University of Defense Technology, Changsha, Hunan province, China

Keywords: Anti-saturation, Anti-jamming, 4-antenna Array, Two-stage.

\begin{abstract}
Antenna array with adaptive beam forming technique is an effect way for GPS receiver to suppress the ambient interference, which, however, would be malfunctioning due to the saturation problem in radio frequency (RF) front or intermediate frequency (IF) front caused by the strong interference. To tackle it, this paper demonstrates a scenario consisting of two stages processing, i.e. two anti-saturation modules in RF-front and one anti-jamming module in IF front. The anti-saturation module based on two antennas, is realized with the RF phase shifter and amplifier and capable of forming a deep null in the direction of the strong interference, while the anti-jamming module employs the blind space time adaptive processing (STAP) algorithm to improve the anti-jamming capability. Compared to the RF-front beam former or the IF-front anti-jamming algorithm, not only can the proposed system achieve the anti-saturation and anti-jamming performance but also has more degrees of freedom to suppress interferences. The feasibility is proved by the experiment, exhibiting at least $21 \mathrm{~dB}$ anti-saturation improvement and nearly $60 \mathrm{~dB}$ interference mitigation.
\end{abstract}

\section{Introduction}

From the google maps and the popular game 'pokemon go' (based on the position of the player), the GPS service is widely used in our daily life. However, the weak power of GPS signal in the receiver varying from -130 to $-110 \mathrm{dBm}$, make it vulnerable to the environmental interference [1]. The adaptive beam forming technique based on antenna array was the dominated method to mitigate the interference, which attracted a lot of researchers' attention in the past decades [2,3,4]. For continuous wave interferences (CWIs), [5] reported an anti-jamming system comprising cascaded CWI-detectable adaptive notch filter (ANF) modules, with each module able to mitigate one CWI. Several windows were employed to increase time-frequency plane resolution in an enhanced short-time Fourier transform (STFT)-based jamming mitigation system [6]. Wavelet packet transform(WPT)-based mitigation blocks were introduced and investigated for anti-jamming in [7]. A two-stage blind adaptive anti-jamming algorithm can cancel interferences by projecting the array received signals on the orthogonal complement space of interferences, which utilized the CLEAN method to estimate direction of arrivals of GPS signals [8]. To improve the anti-jamming capability, a vector delay lock loop (VDLL) is proposed in [9].

The aforementioned approaches are capable of suppressing the ambient interference on the condition of the RF or IF front end is not in saturation. For significantly strong interference, the LNA in the RF front or the amplifier in the IF front would be saturated, 
hence introducing the huge distortion and making those algorithms ineffective. The traditional way to solve the saturation problem is to install the automatic gain control (AGC) either in the RF front or in the IF front, which was able to control the input signal within the linear area of the amplifier. Whereas the low gain of AGC would make the receiver non-sensitive to the GPS signals. Besides, the ratio of the GPS signal to the interference does not have any improvement. Rare study has focus on the saturation problem of the GPS receiver. In this paper, two stages processing based on 4 antennas are proposed to realize the anti-saturation and anti-jamming performance.

\section{System Scheme}

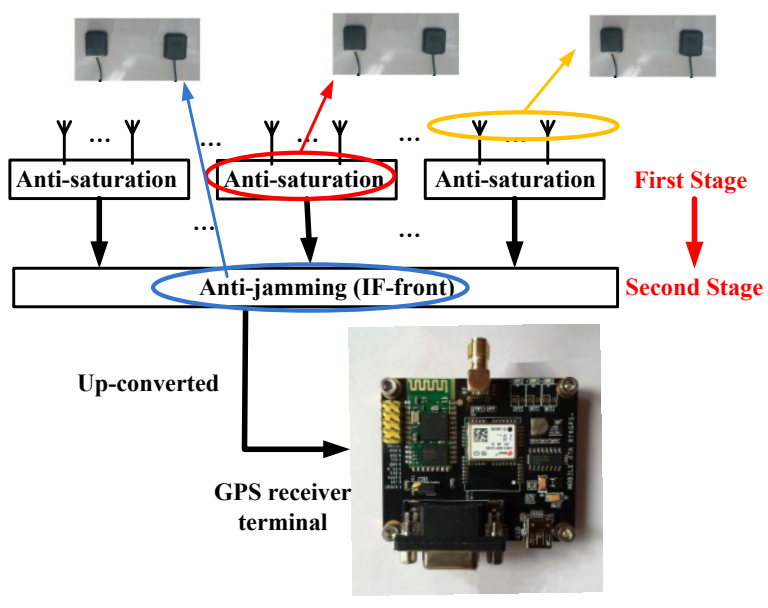

Figure.1 The scheme of two-stage system.

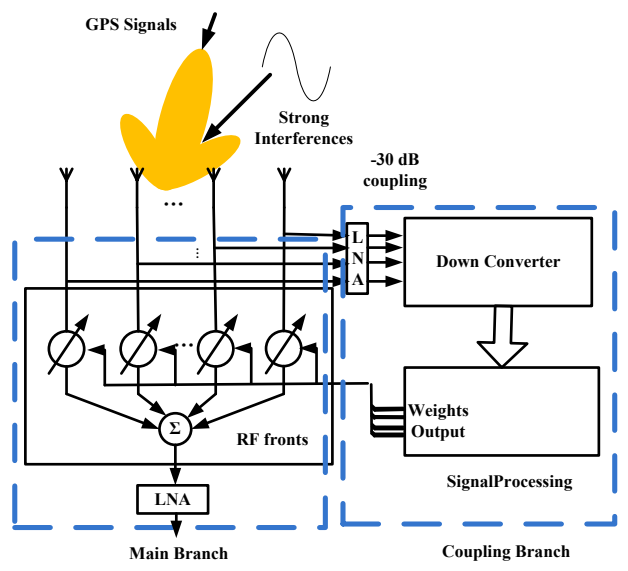

Figure.2 The anti-saturation module.

Fig.1 shows the scheme of proposed two-stage system, including several anti-saturation modules and one anti-jamming module. Each anti-saturation in the first stage, comprised of a GPS sub-array, is realized by traditional Power Inversion (PI) algorithm with the RF phase shifter and amplifier. The detailed scenario of the anti-saturation module is demonstrated in Fig.2. The received signals are firstly divided into two branches, i.e. the main and the coupling branch. Most of the energy passes through the main branch, while $-30 \mathrm{~dB}$ energy is coupled to the coupling branch. For magnificently strong interference, the main branch would be saturated without specific beam forming, while the coupling branch would not suffer from the saturation problem due to the low coupling. PI algorithm can be employed to help update the RF weight in the main 
branch to suppress the strong interference. To make the weights operate well in the main branch, the compensation and calibration of these two branches should be made.

The outputs of the anti-saturation modules are connected to the input array of the anti-jamming module in the second stage, where the blind STAP algorithm was employed in IF front to further suppress the remaining interferences processed after first stage. By increasing the number of taps in STAP, the capability of the anti-jamming can be improved.

Compared to RF beamforming, this two-stage system can have more degrees of freedom to suppress interference, benefiting from the implementation of STAP. In comparison with the IF beamforming, the proposed scenario can solve the saturation problem in RF front. Overall, the two-stage system combining advantages of RF and IF beamforming, can obtain both good anti-saturation and anti-jamming performance. For simplicity and practicality, two anti-saturation modules based on 4-antenna linear array and one anti-jamming module are designed in the practical system. The distance of the adjacent antenna is half wavelength. Since the saturation problem in RF front can be also observed in the IF front, this paper choose the IF front to measure the anti-saturation performance. 3-tap blind STAP realized by FPGA is implemented in the anti-jamming module within the IF bandwidth (30 MHz).

\section{Experiment Results and Analysis}

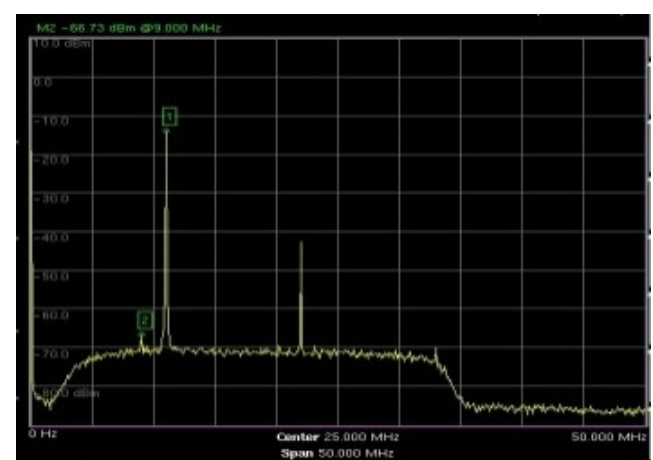

(a)

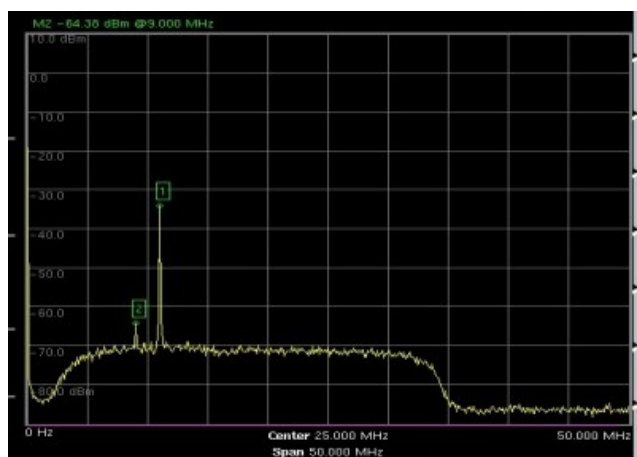

(b)

Figure.3 Observation of signal in IF front. (a)Unprocessed by anti-saturation module(b)Processed by anti-saturation module.

In order to test the effectiveness of the proposed method based on the RF front-end amplitude and phase weighting, a simple verification test is done in this paper. To observe a clear anti-saturation performance, a weak signal working in GPS $L_{1}$ band $(1575 \mathrm{MHz})$ with $-80 \mathrm{dBm}$ power, rather than the existing GPS signal, is generated in the experiment, whereas a strong interference with the power $50 \mathrm{~dB}$ greater than the weak signal is simultaneously transmitted. The direction of arrival weak signal is $\theta=0^{\circ}$, while the strong interference illuminates in $\theta=45^{\circ}$. The strong interference working in $1574 \mathrm{MHz}$ makes the receiver work in the saturation area as shown in the Fig. 3 where marker 1 denotes the interference in IF front. And marker 2 represents the down converted weak signal. The saturation introduces other frequencies and leads the serious distortion into the IF front end. By measuring the power of interference when saturation occurs for being processed and unprocessed by anti-saturation module, the improvement of anti-saturation of this method can be obtained. Processed by the RF anti-saturation module, the strong interference is noticeably suppressed by roughly 21 $\mathrm{dBm}$, while the weak signal witnesses a minor increase from -66 to $-64 \mathrm{dBm}$. No other 
frequencies is introduced into the working band, showing the receiver's RF front operating in linear area instead of the saturation area. The anti-saturation performance for different interference frequencies ranging from $1571 \mathrm{MHz}-1580 \mathrm{MHz}$ is presented in Table 1.

Table 1. Anti-saturation performance

\begin{tabular}{|l|c|c|c|c|c|}
\hline Frequency (/MHz) & 1571 & 1572 & 1573 & 1574 & 1575 \\
\hline Anti-saturation (dB) & 21 & 21 & 20 & 21 & 21 \\
\hline Frequency (/MHz) & 1576 & 1577 & 1578 & 1579 & 1580 \\
\hline Anti-saturation (dB) & 24 & 22 & 23 & 25 & 23 \\
\hline
\end{tabular}

Table 2. Total anti-jamming performance

\begin{tabular}{|l|c|c|c|c|c|}
\hline Frequency (/MHz) & 1571 & 1572 & 1573 & 1574 & 1575 \\
\hline Anti-jamming (dB) & 59 & 57 & 58 & 61 & 60 \\
\hline Frequency (/MHz) & 1576 & 1577 & 1578 & 1579 & 1580 \\
\hline Anti-jamming (dB) & 59 & 60 & 58 & 61 & 59 \\
\hline
\end{tabular}

The response time of the anti-saturation module is another factor needs to be evaluated. For moving interference target, the long response time of anti-saturation would affect the performance, since the interference is not in the direction that the null of beams steer. Given that the RF and IF signals are similar and the response time is the same by the proposed method, the oscilloscope working in IF band is used for measurement. The oscilloscope is configured with single trigger to capture response processing when the power source of the strong interference is switched on. The former dashed line in Fig.4 illustrates the start of the interference signal, while the latter dashed line shows the time when the interference is suppressed into a steady level. By measuring the time distance of these two time lines, the response time (47us) of the anti-saturation module can be obtained, which is significantly short compared to the speed of the moving interference target.

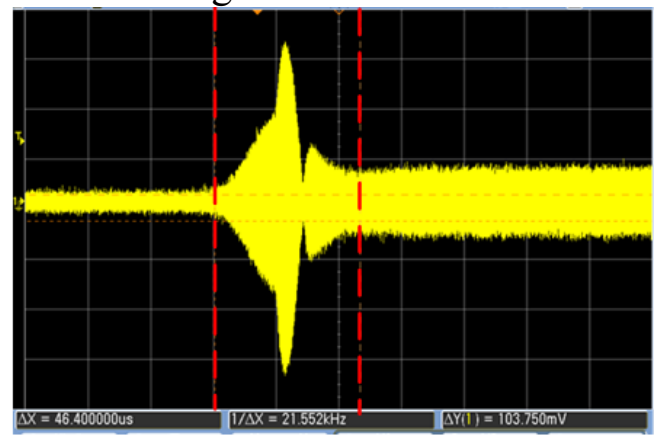

Figure.4 The response time of the anti-saturation module.

After first stage processing by anti-saturation modules, the GPS signal together with the suppressed interference are fed into the second stage i.e. the anti-jamming module. To measure the anti-jamming performance, the IF signal is up-converted to the GPS receiver terminal. The total anti-jamming performance can be obtained by changing the power of the interference until the terminal loses the function to locate the position. The interference mitigation of the proposed two-stage scheme with 4-antenna is listed in 
Table 2. Around $60 \mathrm{~dB}$ anti-jamming performance can be observed for interference ranging from $1570 \mathrm{MHz}$ to $1580 \mathrm{MHz}$.

\section{Conclusion and Discussion}

For strong interference leading the saturation problem for RF or IF front of the GPS receiver, this paper elaborated a two-stage scheme to realize the adaptive anti-saturation and anti-jamming performance. The first stage consists of two RF-front beamformers, with each beamformer comprised of two antennas. The aim of the first stage is to suppress the strong interference in a low power level, while the second stage utilizing the blind STAP in IF front is to increase the capability of the anti-jamming. In comparison with the RF or IF beamforming, the two-stage module can not only tackle the saturation problem but also achieve a high-capability anti-jamming performance. The system with 4 antennas was experimentally shown to have at least $21 \mathrm{~dB}$ anti-saturation performance with only 47 us response time, and around $60 \mathrm{~dB}$ anti-jamming improvement for the receiver. Other systems with more antenna elements and taps will be studied in the further researches to investigate the anti-saturation and anti-jamming performance.

\section{References}

[1] Dovis, Fabio.: 'GNSS Interference Threats and Countermeasures', Artech House, 2015.

[2] Gao, G.X., Heng, L., Hornbostel, A., Denks, H., Meurer, M., Walter, T. and Enge, P.: 'DME/TACAN interference mitigation for GNSS: algorithms and flight test results', GPS solutions, 2013, 17(4), pp 561-573.

[3] Sgammini, M., Antreich, F., Kurz, L., Meurer, M. and Noll, T.G.: 'Blind adaptive beamformer based on orthogonal projections for GNSS', 2012.

[4] Mañosas-Caballú, M., Seco-Granados, G. and Swindlehurst, A.L.: 'Robust beamforming via FIR filtering for GNSS multipath mitigation', In2013 IEEE International Conference on Acoustics, Speech and Signal Processing .

[5] Chien, Y.R.: 'Design of GPS anti-Jamming systems using adaptive notch filters', IEEE Systems Journal, 2015, 9(2), pp 451-460.

[6] Rezaei, M.J., Abedi, M. and Mosavi, M.R.: 'New GPS anti-jamming system based on multiple short-time Fourier transform', IET Radar, Sonar \& Navigation, 2016, 10(4), pp 807-815.

[7] Mosavi, M.R., Rezaei, M.J., Pashaian, M. and Moghaddasi, M.S.:' A fast and accurate anti-jamming system based on wavelet packet transform for GPS receivers.', GPS Solutions,2016, pp 1-12.

[8] Lu, D., Wu, R. and Liu, H.: 'Global positioning system anti-jamming algorithm based on period repetitive CLEAN', IET Radar, Sonar \& Navigation,2013, 7(2), pp 164-169.

[9] Li, Q., Xu, D., Wang, W., Wang, X. and Han, Z.: 'Anti-jamming scheme for GPS receiver with vector tracking loop and blind beamformer', Electronics Letters, 2014,50(19), pp1386-1388. 